Journal of Advanced Research in Fluid Mechanics and Thermal Sciences

Journal homepage: www.akademiabaru.com/arfmts.html ISSN: $2289-7879$

\title{
Convective Boundary Conditions Effect on Cylindrical Media with Transient Heat Transfer
}

\author{
Raoudha Chaabane ${ }^{1,}{ }^{*}$, Nor Azwadi Che Sidik ${ }^{2}$, Abdelmajid Jemni $^{1}$ \\ Laboratory of Thermal and Energetic Systems Studies (LESTE), National School of Engineering of Monastir, University of Monastir, Tunisia \\ 2 Malaysia-Japan International Institute of Technology (MJIIT), Universiti Teknologi Malaysia, Jalan Sultan Yahya Petra, 54100 Kuala Lumpur, \\ Malaysia
}

\begin{tabular}{|c|c|}
\hline ARTICLE INFO & ABSTRACT \\
\hline $\begin{array}{l}\text { Keywords: } \\
\text { Axisymmetric; convective; heat transfer; } \\
\text { boundary condition; Lattice Boltzmann } \\
\text { method }\end{array}$ & $\begin{array}{l}\text { Lattice Boltzmann method is used to solve inside a cylindrical cavity with convective } \\
\text { boundary condition is highlighted in this paper. Because of its simple, stable, accurate, } \\
\text { efficient and ease for parallelization, we use the thermal Single Relaxation Time } \\
\text { Bhatnagar Gross Krook (SRT BGK) mesoscopic approach in order to solve the energy } \\
\text { equation. Thermal fields are simulated using D2Q9 scheme. We introduce and } \\
\text { demonstrate numerically some usual cases (Dirichlet, Newmann) of Boundary } \\
\text { conditions (Bcs). After validation, we extend the present work to the convective case. } \\
\text { At the wall of the cavity, the unknown Thermal Distribution Functions (TDF) are } \\
\text { exposed to the bounce back concept which is determined consistently by one of the } \\
\text { imposed BCs. An in-house Fortran } 90 \text { code is used to analyze a variety of BCs inside a } \\
\text { two-dimensional cavity. In validation, obtained results highlight a good agreement } \\
\text { with literature. The present study is extended to deal with convective boundary } \\
\text { condition for conduction transfer problems inside an axisymmetric cylindrical media } \\
\text { subjected to heat generation and Newman boundary conditions. }\end{array}$ \\
\hline
\end{tabular}

\section{Introduction}

Countless physical models in the engineering applied science are simulated using nonlinear boundary conditions (BCs) value problems where it is usually difficult to obtain the exact analytical solution. Due of this difficulty, most of the researchers in the engineering and the applied sciences directly resort to the numerical codes in order to solve their physical models [1]. However, many other authors investigated their physical models by using analytical or semi-analytical methods [2-7]. Unfortunately, the accuracy of the numerical solutions derived from these analytical or semianalytical methods cannot be checked without addressing the convergence issue.

In this paper, we use LBM to simulate convective boundary condition inside a cylindrical media. In fact, nowadays LBM is considered as bright numerical technique for simulating thermal and fluid flows associated with complex boundary conditions [8-20]. it is known that conventional numerical methods (CFD) discretize the macroscopic equations. However, LBM is based on simplified kinetic

\footnotetext{
* Corresponding author.

E-mail address: raoudhach@gmail.com
}

https://doi.org/10.37934/arfmts.82.2.146156 
models integrating physics of microscopic processes where macroscopic properties obey the desired equations.

In the last decade, LBM was used to simulate heat transfer in different engineering applications such as thermal insulation, heat pipes, cooling of electronic components cooling, solar collectors (SC), heat exchangers, solar dryers air heating systems, storage technology, passive solar heating [21-26]. Owing to their accuracy and simplicity for studying confining cavities with mixed BCS, LBM is the suitable numerical choice when dealing with complex BCs.

In literature, few publications deal with LB studies that consider mixed Boundary conditions as Dirichlet, Newman, convective Boundary conditions. In validation, we consider two test cases for the solution of conduction problems in Cartesian geometry involving Dirichlet BCs and volumetric heat generation effect. For validation, two test benchmark problems are studied and good agreement is obtained. The present numerical approach can cope with more complex geometry and, thus, it will be particularly efficient in resolving transient conduction in axisymmetric cavity in the presence of heat generation effect with mixed boundary condition.

\section{Numerical Approach}

Heat transfer inside rectangular and axisymmetric enclosure is considered. Thermo-physical properties of the medium are assumed constant. For the problem under consideration, and in the absence of convection and radiation, the energy equation is given by

$$
\frac{\partial T}{\partial t}=\alpha \nabla^{2} T+Q
$$

because the LBM is based on the discrete Boltzmann kinetic equation, heat transport problems are solved using the internal energy evolution equation of the nine-speed (D2Q9) Lattice Boltzmann model is given by [27-29]

$$
\frac{\partial f_{i}(\vec{r}, t)}{\partial t}+\vec{e}_{i} \nabla f_{i}(\vec{r}, t)=\Omega_{i}, \quad i=1,2,3, \ldots \ldots b
$$

where,

$b$ is the number of directions in a D2Q9 lattice

$\Omega_{i}$ is the collision operator defined as

$$
\Omega_{i}=-\frac{1}{\tau}\left[f_{i}(\vec{r}, t)-f_{i}^{(0)}(\vec{r}, t)\right]
$$

$f_{i}$ is the particle distribution function denoting the number of particles at the lattice node $\vec{r}$ at time $\mathrm{t}$ moving in direction $i$ with velocity $\overrightarrow{e_{i}}$ along the lattice link $\Delta \vec{r}=\overrightarrow{e_{i}} \Delta t$ connecting the nearest neighbours (Figure 1).

The temperature $T(\vec{r}, t)$ is defined as

$$
T(\vec{r}, t)=\sum_{i=0}^{b} f_{i}(\vec{r}, t)
$$

where the observed flux is expressed by 
$\sum_{i=0}^{b} f_{i}(\vec{r}, t) \vec{e}_{i}$

In BGK model, the discrete evolution equation is given as

$\frac{\partial f_{i}(\vec{r}, t)}{\partial t}+\overrightarrow{e_{i}} \nabla f_{i}(\vec{r}, t)=-\frac{1}{\tau}\left[f_{i}(\vec{r}, t)-f_{i}^{(0)}(\vec{r}, t)\right]$

$f_{i}^{(0)}$ is the equilibrium distribution function.

Based on SRT model, the relaxation time can be related with the thermal diffusivity $\alpha$, the lattice velocity $C$ and the time step $\Delta t$ by the following relation [29]

$\tau=\frac{3 \alpha}{|C|^{2}}+\frac{\Delta t}{2}$

The nine velocities $\overrightarrow{e_{i}}$ in the D2Q9 (Figure 1) and their corresponding weights $w_{i}$ are the following

$\vec{e}_{0}=(0,0)$

$\vec{e}_{i}=\left(\cos \left(\varphi_{i}\right), \sin \left(\varphi_{i}\right)\right) \cdot C$

for $\varphi_{i}=(i-1) \pi / 2 \quad i=1,2,3,4$

$\vec{e}_{i}=\sqrt{2}\left(\cos \left(\varphi_{i}\right), \sin \left(\varphi_{i}\right)\right) \cdot C$

for $\varphi_{i}=(i-5) \pi / 2+\pi \quad i=5,6,7,8$

$w_{i}=\frac{4}{9}$

$w_{i}=\frac{1}{9}$ for $i=1,2,3,4$

$w_{i}=\frac{1}{36}$ for $i=5,6,7,8$

In the presence of volumetric heat generation, Eq. (6) can be written as

$$
\begin{aligned}
f_{i}\left(\vec{r}+\vec{e}_{i} \Delta t, t+\Delta t\right) & =f_{i}(\vec{r}, t)-\frac{\Delta t}{\tau}\left[f_{i}(\vec{r}, t)-f_{i}^{(0)}(\vec{r}, t)\right] \\
& +w_{i} \Delta t Q^{*}
\end{aligned}
$$

where,

$Q^{*}$ is the non-dimensional heat generation.

For heat conduction problems, the equilibrium distribution function is given as

$f_{i}^{(0)}(\vec{r}, t)=w_{i} T(\vec{r}, t)$ 
In LBM boundary condition, BCs properties of the known and unknown populations on each side as shown on Figure 1.

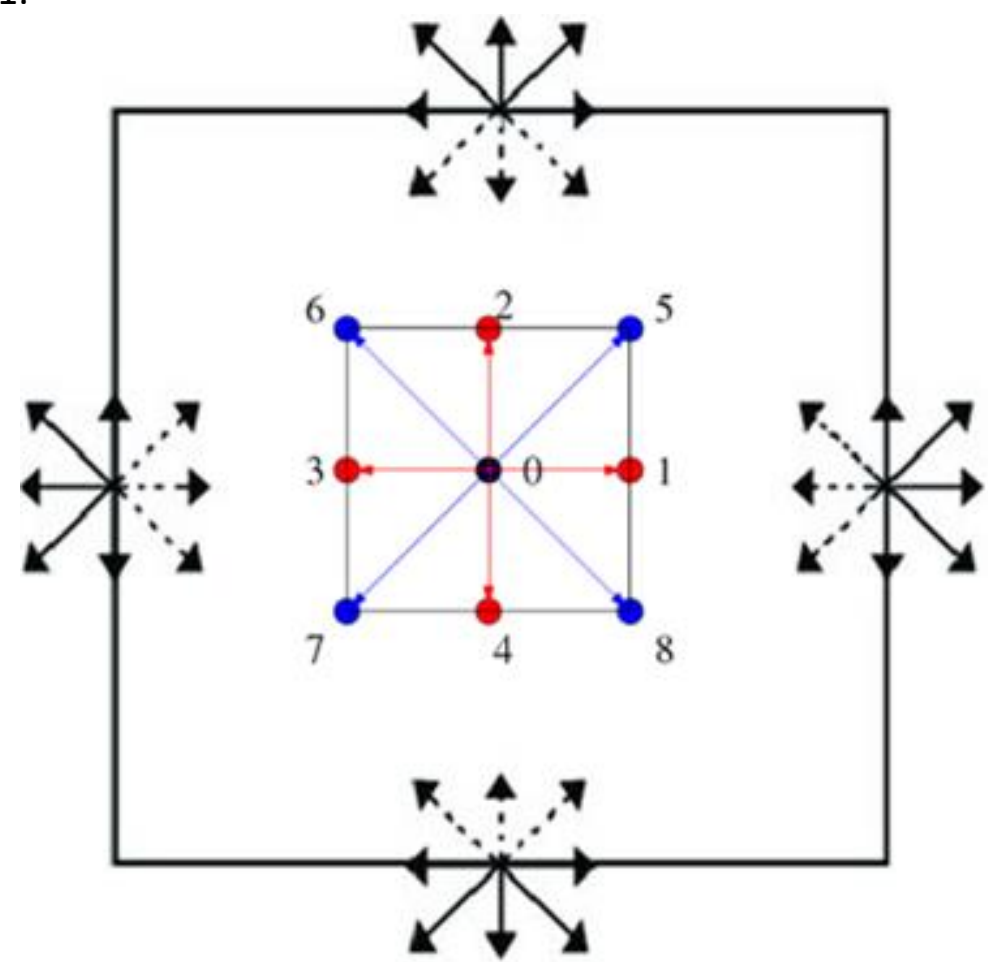

Fig. 1. BGK D2Q9 LBM Basic lattice and known and unknown populations in the computational domain

\section{Results and Discussion}

For validation, we deal with transient heat conduction problems in two-dimensional Cartesian enclosure with the four boundaries at isothermal temperatures. Initial and the boundary conditions are written as

Initial condition

$T(x, y, 0)=T_{r e f}$

Boundary conditions

$T(x, 0, t)=0.25 T_{r \mathrm{e} f}$

$T(x, Y, t)=T(0, y, t)=T(X, y, t)=T_{r e f}$

For this case, $\xi=\alpha t / L^{2}$ is the non-dimensional time and $L$ is the characteristic length. $\Delta \xi$ was taken as $10^{-4}$. Besides, transient and steady state conditions were highlighted. Convergence criteria assume that temperature difference between two consecutive time levels at each lattice centre did not exceed $10^{-6}$. The same configuration is simulated, also, using the Finite Volume Method (FVM). The two numerical results are compared with literature. Figure 2 display non dimensional centreline $(\mathrm{x} / \mathrm{X}=0.5$ ) temperature at different instants $\xi$ and good concordance is highlighted. 


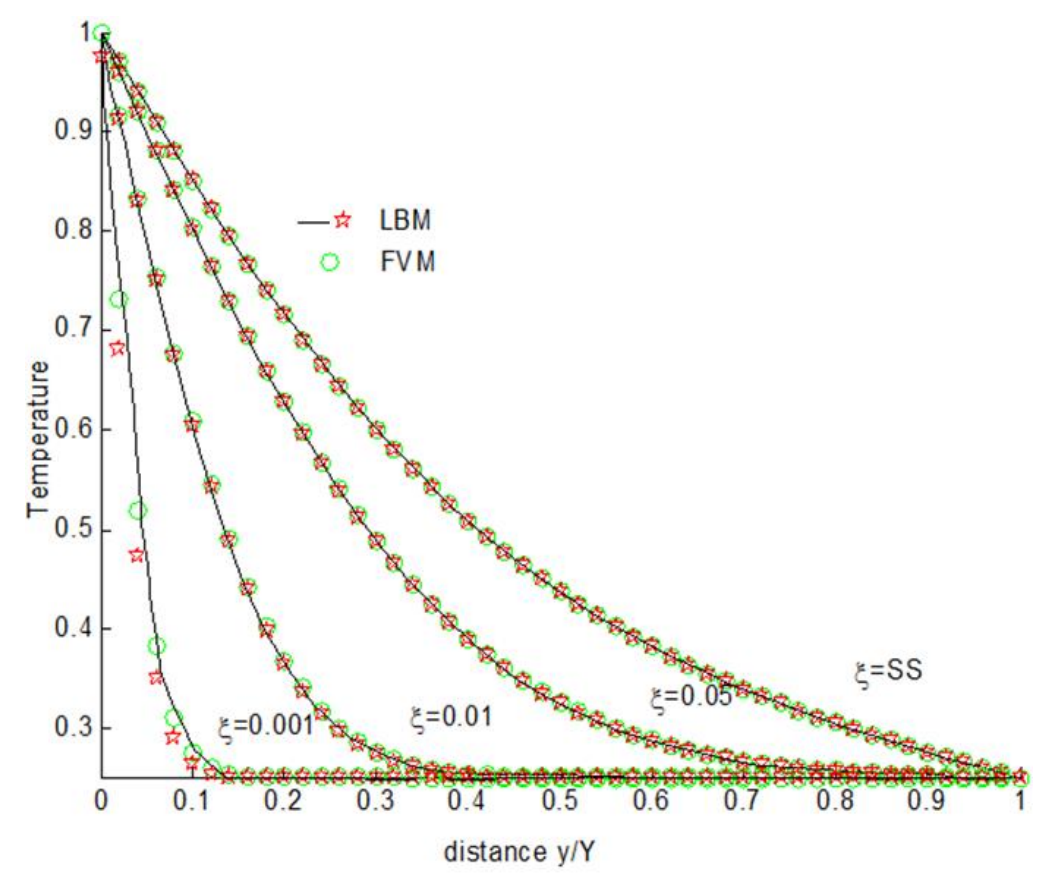

Fig. 2. Dimensionless mid-plane $(x / X=0.5)$ temperature evolution for different dimensionless instants

Then, the effect of volumetric heat generation is studied. With a unity non dimensional volumetric heat generation, the four walls of the Cartesian cavity are subjected to a specified temperature (Dirichlet boundary condition). We notice that effect of heat generation is very less in the beginning compared to steady state because it takes some time to influence the temperature profile.

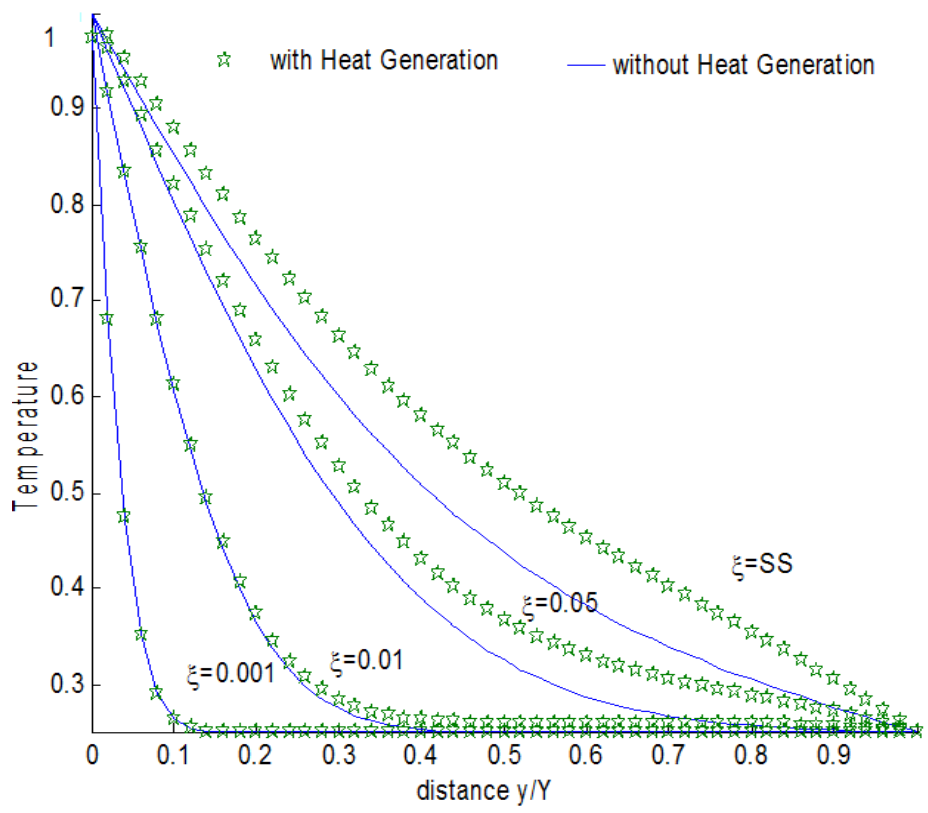

Fig. 3. Effect of volumetric heat generation on dimensionless mid-plane $(x / X=0.5)$ temperature

This section deals with transient heat conduction equation in cylindrical coordinates. When Heat conduction in an infinite solid with a long cylindrical cavity is discussed, axisymmetric treatment is investigated. For this case (Figure 4), the system is initially at temperature 


$$
T(x, y, 0)=T_{0}
$$

For time $t>0$, the bottom and top boundaries are at prescribed fluxes, respectively.

$$
\begin{aligned}
& \frac{\partial T}{\partial y}=Q_{S} \\
& -\frac{\partial T}{\partial y}=Q_{N}
\end{aligned}
$$

For the physical problem addressed in this section (Figure 4), the thermal boundary condition at the lateral surface is subjected to convective heat flux boundary condition with non dimensional volumetric heat generation $\left(Q^{*}=2\right)$ written as

$$
-k \frac{\partial T}{\partial x}=Q+h\left(T-T_{\infty}\right)
$$

where,

$h$ is the convective heat transfer coefficient.

$x, y$ is the direction of outward normal to the surface concerned.

$k$ Thermal conductivity

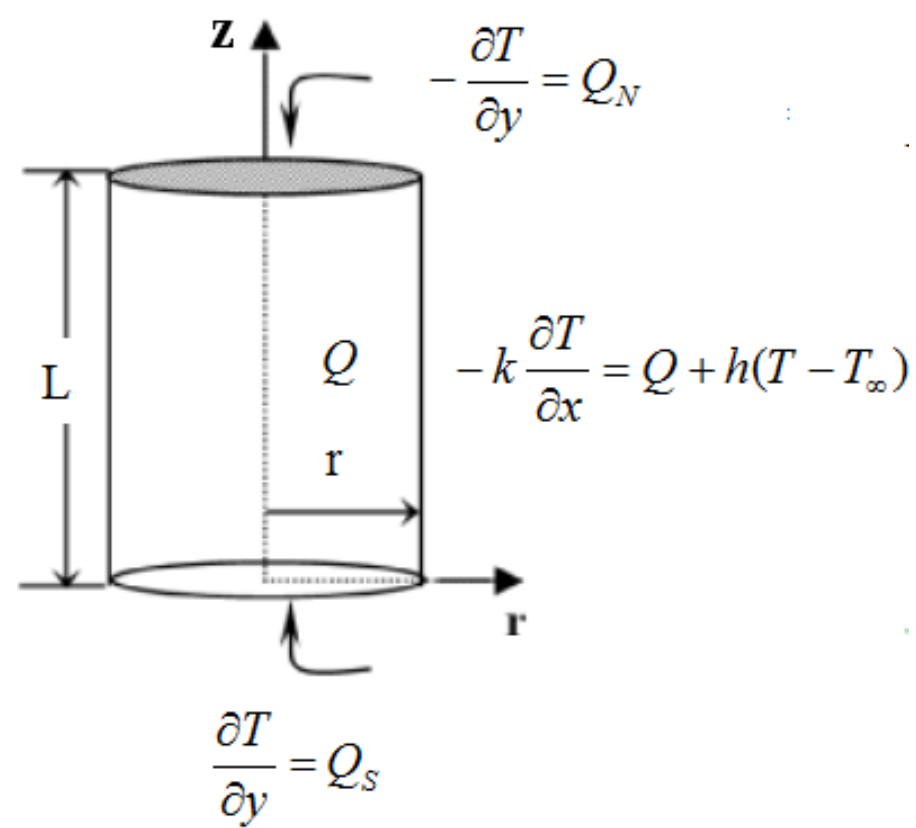

Fig. 4. Cylindrical boundary conditions

In non-dimensional equations model, we define Biot number as

$$
B i=\frac{h L}{k}
$$


The distributions of isotherms inside cylindrical media are plotted in the Figure 5 for different where the axisymmetric is subjected to a volumetric heat generation condition $\left(Q^{*}=2\right)$ with Biot number of 20 in a $100 \times 100$ grid (Figure 5).
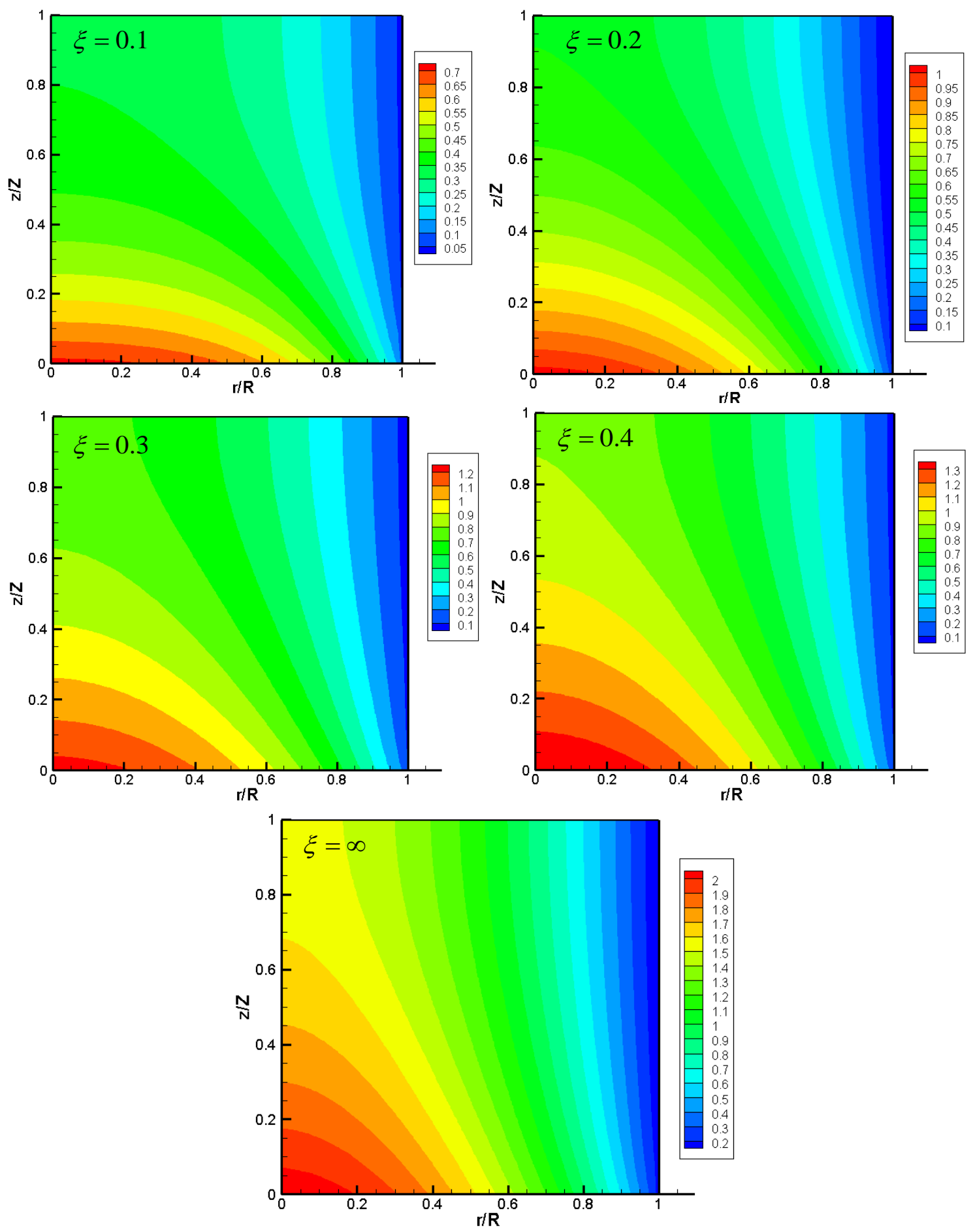

Fig. 5. Transient dimensionless isotherms in the presence of non-dimensional volumetric heat generation source $\left(Q^{*}=2\right)$ for different instants $\xi$ (Cylindrical case) 
In order to analyze the effect of the non-dimensional Biot number, steady state non dimensional temperature radial (Figure 6) and axial (Figure 7) is plotted for the case of axisymmetric geometry with BCs of Figure 4. We notice that radial and axial are more enhanced in presence of smallest Biot number.

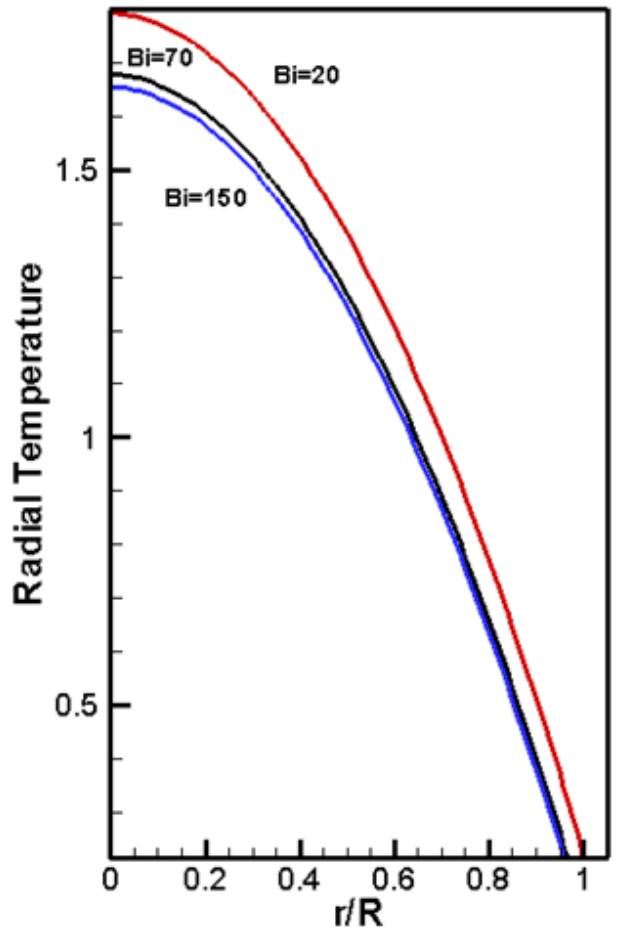

Fig. 6. Steady state dimensionless radial temperatures in the presence of nondimensional volumetric heat generation source $\left(Q^{*}=2\right)$ for different Biot numbers (Cylindrical case)

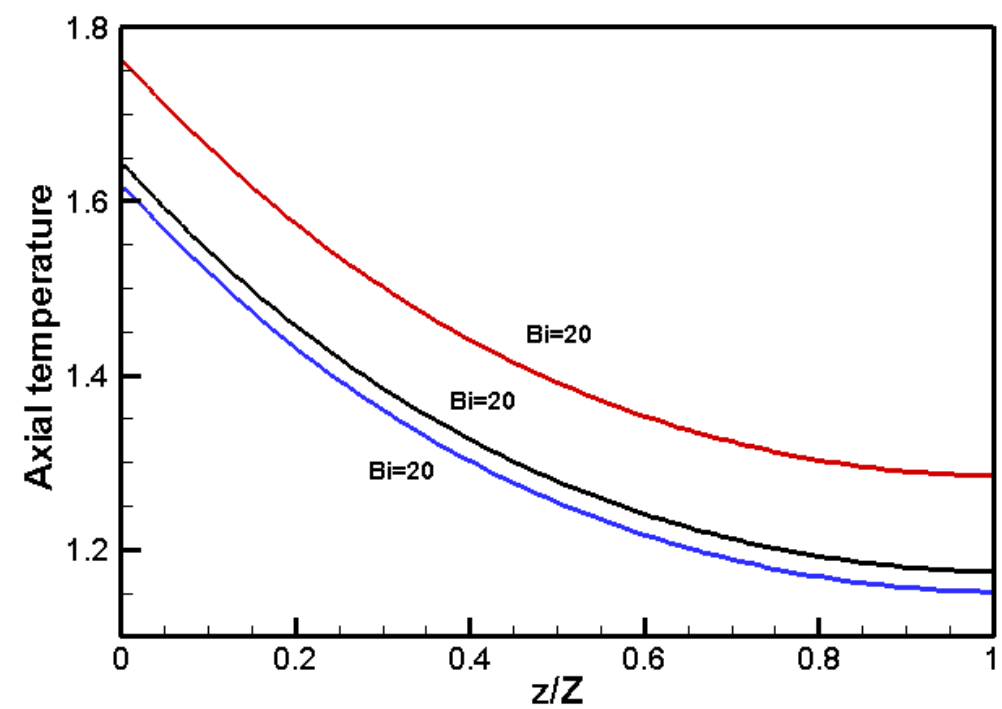

Fig. 7. Steady state dimensionless axial temperatures in the presence of non-dimensional volumetric heat generation source $\left(Q^{*}=2\right)$ for different Biot numbers (Cylindrical case) 
Because LBM is time varying mesoscopic approach, we highlight in Figure 8, the time evolution of the dimensionless temperature at $(r / 2 R, z / 2 Z)$. We notice that temperature increases with time. The steady state temperature is reached at roughly time $=15000$.

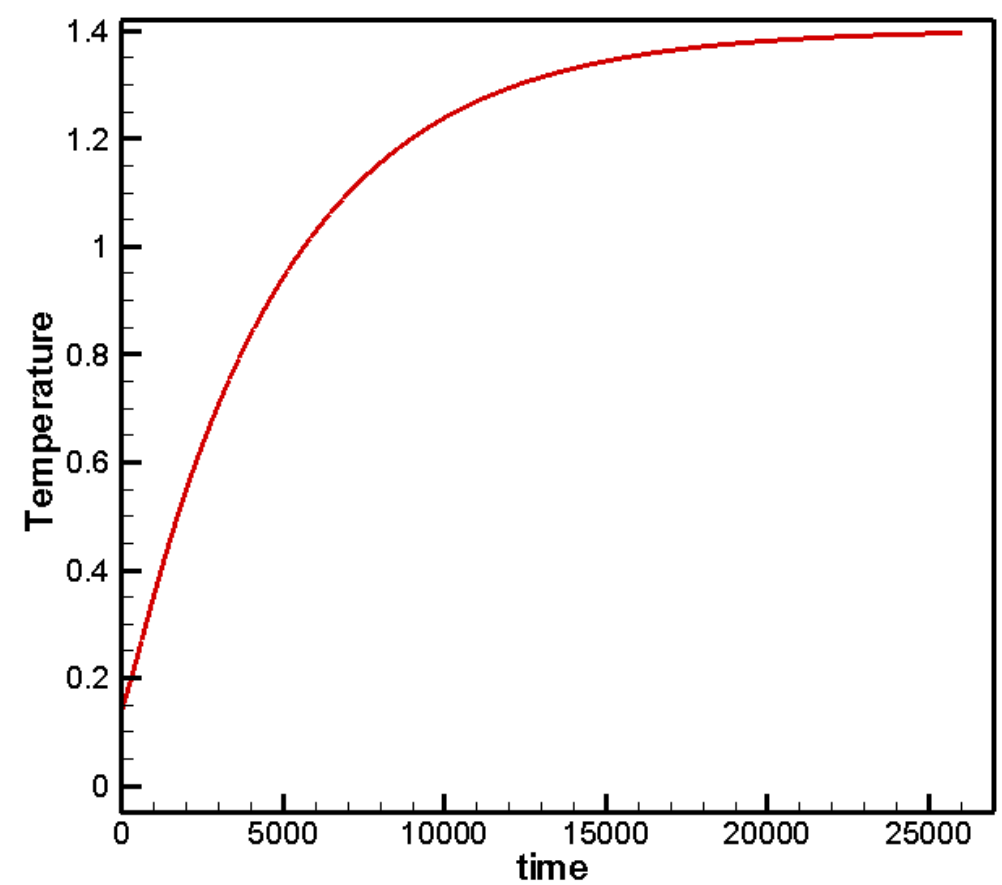

Fig. 8. Time evolution of the temperature at the centerline of the axisymmetric cavity

\section{Conclusions}

After good validation, LBM is extended to simulate numerically a complex geometry with mixed $\mathrm{BCs}$ in order to solve transient heat conduction problems in cylindrical media in the presence of heat generation effect subjected to convective boundary condition in lateral surface. Bottom wall is subjected to hot heat flux and top one is at cold heat flux. The present code shows a great stability to deal with different sets of mixed BCs; namely constant temperature, flux boundary conditions and convective boundary conditions. The effect of volumetric heat source in the cylindrical cavity is highlighted. The present numerical code achieves an efficient stability, that make it a crucial future tool to predict heat transfer and fluid flow characteristics for convection radiation engineering energy problems

\section{References}

[1] Ebaid, Abdelhalim. "A new analytical and numerical treatment for singular two-point boundary value problems via the Adomian decomposition method." Journal of Computational and Applied Mathematics 235, no. 8 (2011): 19141924. https://doi.org/10.1016/j.cam.2010.09.007

[2] Adomian, G. "Solving frontier problems of physics: the decomposition method, With a preface by Yves Cherruault." Fundamental Theories of Physics, Kluwer Academic Publishers Group, Dordrecht 1 (1994). https://doi.org/10.1007/978-94-015-8289-6

[3] Aly, Emad H., Abdelhalim Ebaid, and Randolph Rach. "Advances in the Adomian decomposition method for solving two-point nonlinear boundary value problems with Neumann boundary conditions." Computers \& Mathematics with Applications 63, no. 6 (2012): 1056-1065. https://doi.org/10.1016/i.camwa.2011.12.010

[4] Ebaid, Abdelhalim. "Analytical Solutions for the Mathematical Model Describing the Formation of Liver Zones via Adomian's Method." Computational \& Mathematical Methods in Medicine (2013). https://doi.org/10.1155/2013/547954 
[5] Ebaid, Abdelhalim. "Approximate analytical solution of a nonlinear boundary value problem and its application in fluid mechanics." Zeitschrift für Naturforschung A 66, no. 6-7 (2011): 423-426. https://doi.org/10.1515/zna-2011$\underline{6-707}$

[6] Wazwaz, Abdul-Majid, Randolph Rach, and Jun-Sheng Duan. "Adomian decomposition method for solving the Volterra integral form of the Lane-Emden equations with initial values and boundary conditions." Applied Mathematics and Computation 219, no. 10 (2013): 5004-5019. https://doi.org/10.1016/i.amc.2012.11.012

[7] McNamara, Guy R., and Gianluigi Zanetti. "Use of the Boltzmann equation to simulate lattice-gas automata." Physical Review Letters 61, no. 20 (1988): 2332. https://doi.org/10.1103/PhysRevLett.61.2332

[8] Higuera, F. J., and Javier Jiménez. "Boltzmann approach to lattice gas simulations." EPL (Europhysics Letters) 9 , no. 7 (1989): 663. https://doi.org/10.1209/0295-5075/9/7/009

[9] Khan, Ansab Azam, Khairy Zaimi, Suliadi Firdaus Sufahani, and Mohammad Ferdows. "MHD Flow and Heat Transfer of Double Stratified Micropolar Fluid over a Vertical Permeable Shrinking/Stretching Sheet with Chemical Reaction and Heat Source." Journal of Advanced Research in Applied Sciences and Engineering Technology 21, no. 1 (2020): 1-14.

[10] Mishra, Subhash C., Bittagopal Mondal, Tanuj Kush, and B. Siva Rama Krishna. "Solving transient heat conduction problems on uniform and non-uniform lattices using the lattice Boltzmann method." International Communications in Heat and Mass Transfer 36, no. 4 (2009): $322-328$. https://doi.org/10.1016/j.icheatmasstransfer.2009.01.001

[11] Bakar, Norhaliza Abu, and Rozaini Roslan. "Mixed Convection in a Lid-Driven Horizontal Cavity in the Presence of Internal Heat Generation or Absorption." Journal of Advanced Research in Numerical Heat Transfer 3, no. 1 (2020): 1-11.

[12] Wolf-Gladrow, Dieter A. Lattice-Gas Cellular Automata and Lattice Boltzmann Models: An Introduction. Springer Science \& Business Media, 2000. https://doi.org/10.1007/b72010

[13] Succi, Sauro, Roberto Benzi, and Francisco Higuera. "The lattice Boltzmann equation: a new tool for computational fluid-dynamics." Physica D: Nonlinear Phenomena 47, no. 1-2 (1991): 219-230. https://doi.org/10.1016/01672789(91)90292-H

[14] Sahak, Ahmad Sofianuddin A., Nor Azwadi Che Sidik, Siti Nurul Akmal Yusof, and Mahmoud Ahmed Alamir. "Numerical Study of Particle Behaviour in a Mixed Convection Channel Flow with Cavity using Cubic Interpolation Pseudo-Particle Navier-Stokes Formulation Method." Journal of Advanced Research in Numerical Heat Transfer 1, no. 1 (2020): 32-51.

[15] Kang, Qinjun, Dongxiao Zhang, and Shiyi Chen. "Unified lattice Boltzmann method for flow in multiscale porous media." Physical Review E 66, no. 5 (2002): 056307. https://doi.org/10.1103/PhysRevE.66.056307

[16] Schure, Mark R., Robert S. Maier, Daniel M. Kroll, and H. Ted Davis. "Simulation of packed-bed chromatography utilizing high-resolution flow fields: comparison with models." Analytical Chemistry 74, no. 23 (2002): 6006-6016. https://doi.org/10.1021/ac0204101

[17] Kandhai, Drona, Dzmitry Hlushkou, Alfons G. Hoekstra, Peter MA Sloot, H. Van As, and U. Tallarek. "Influence of stagnant zones on transient and asymptotic dispersion in macroscopically homogeneous porous media." Physical Review Letters 88, no. 23 (2002): 234501. https://doi.org/10.1103/PhysRevLett.88.234501

[18] Sahak, Ahmad Sofianuddin A., Nor Azwadi Che Sidik, and Siti Nurul Akmal Yusof. "Cubic Interpolation PseudoParticle Navier-Stokes Formulation Method for Solid Particle-Fluid Interaction in Channel Flow with Cavity." Journal of Advanced Research in Materials Science 70, no. 1 (2020): 1-17.

[19] Drazer, German, and Joel Koplik. "Tracer dispersion in two-dimensional rough fractures." Physical Review E 63, no. 5 (2001): 056104. https://doi.org/10.1103/PhysRevE.63.056104

[20] Maier, Robert S., Daniel M. Kroll, H. Ted Davis, and Robert S. Bernard. "Simulation of flow in bidisperse sphere packings." Journal of Colloid and Interface Science 217, no. 2 (1999): $341-347$. https://doi.org/10.1006/jcis.1999.6372

[21] Ho, Jeng-Rong, Chun-Pao Kuo, Wen-Shu Jiaung, and Cherng-Jyh Twu. "Lattice Boltzmann scheme for hyperbolic heat conduction equation." Numerical Heat Transfer: Part B: Fundamentals 41, no. 6 (2002): 591-607. https://doi.org/10.1080/10407790190053798

[22] Bekri, S., J. Howard, J. Muller, and P. M. Adler. "Electrical resistivity index in multiphase flow through porous media." Transport in Porous Media 51, no. 1 (2003): 41-65. https://doi.org/10.1023/A:1021229106005

[23] Zeiser, Thomas, Martin Steven, Hannsjörg Freund, Peter Lammers, Gunther Brenner, Franz Durst, and Jörg Bernsdorf. "Analysis of the flow field and pressure drop in fixed-bed reactors with the help of lattice Boltzmann simulations." Philosophical Transactions of the Royal Society of London Series A 360, no. 1792 (2002): 507-520. https://doi.org/10.1098/rsta.2001.0945 
[24] Bhatnagar, Prabhu Lal, Eugene P. Gross, and Max Krook. "A model for collision processes in gases. I. Small amplitude processes in charged and neutral one-component systems." Physical Review 94, no. 3 (1954): 511. https://doi.org/10.1103/PhysRev.94.511

[25] Chen, Shiyi, Hudong Chen, Daniel Martnez, and William Matthaeus. "Lattice Boltzmann model for simulation of magnetohydrodynamics." Physical Review Letters 67, no. $27 \quad$ (1991): 3776. https://doi.org/10.1103/PhysRevLett.67.3776

[26] Yoshino, Masato, and Takaji Inamuro. "Lattice Boltzmann simulations for flow and heat/mass transfer problems in a three-dimensional porous structure." International Journal for Numerical Methods in Fluids 43, no. 2 (2003): 183198. https://doi.org/10.1002/fld.607

[27] Qian, Yue-Hong, Dominique d'Humières, and Pierre Lallemand. "Lattice BGK models for Navier-Stokes equation." EPL (Europhysics Letters) 17, no. 6 (1992): 479. https://doi.org/10.1209/0295-5075/17/6/001

[28] He, Xiaoyi, and Li-Shi Luo. "Theory of the lattice Boltzmann method: From the Boltzmann equation to the lattice Boltzmann equation." Physical Review E 56, no. 6 (1997): 6811. https://doi.org/10.1103/PhysRevE.56.6811 